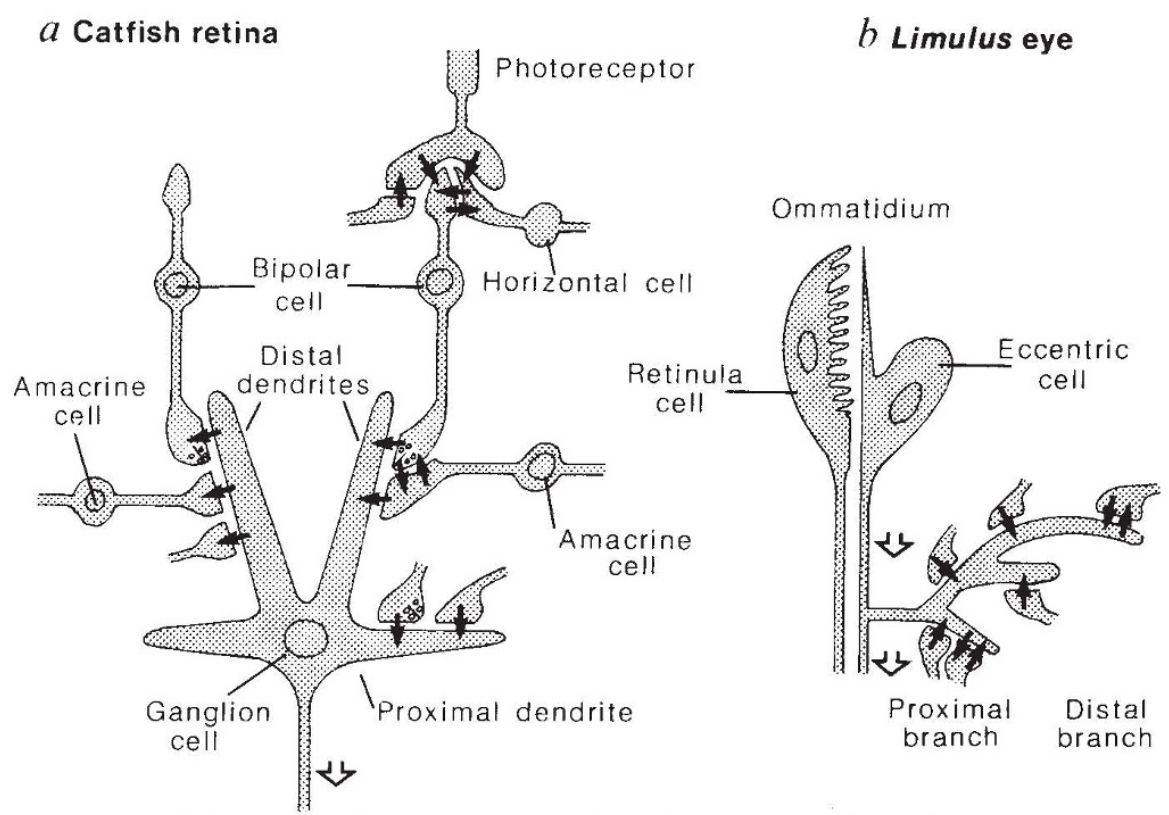

Aspects of the organization of presynaptic dendrites and microcircuits in vertebrate and invertebrate eyes. The sites and polarities of individual chemical synapses are indicated by solid arrows, the direction of spike conduction by open-headed arrows. $a$, Catfish retina: the main types of retinal cells are shown, with some of the main synaptic connections. The connections shown on the right of the ganglion cell are the well-established inputs; on the left, the newly described ganglion cell output synapses on to bipolar cell processes and amacrine or ganglion cell processe ${ }^{3}$, found on distal dendrites of ganglion cells (see text). $b$, Compound eye of Limulus: schematic representation of an ommatidium containing retinular cells and an eccentric cell. The eccentric cell is analogous to the ganglion cell in having a long axon that projects to higher visual centres. The synaptic connections made by a branch in the neural plexus are indicated. There is a predominance of input synapses on proximal branches and a relatively larger proportion of output synapses distally. The significance of this distribution is discussed in the text.

neuronal branches thus seems to be a common mechanism in arthropods.

The ganglion cell is known to lack recurrent axon collaterals and has been believed to function entirely as a follower cell, driven by its inputs from bipolar and amacrine cells. The new evidence ${ }^{3}$ indicates that at least some of the ganglion cells can take part in shaping their own response properties through the microcircuits formed by their presynaptic den- drites. Sakai et al. point out that the synaptic clusters around distal dendrites can function as relatively independent sites for local signal processing, which also seems to apply to the clusters in Limulus. Individual or clustered synapses functioning as integrative units are becoming familiar in many systems ${ }^{9-11}$. The degree to which the relative independence of such units is influenced by passive membrane properties and by active dendritic sites is under investigation in several laboratories.

How are dendritic input and output synapses laid down during ontogeny? This is the subject of recent work by Nishimura and Rakic ${ }^{12}$ in the inner plexiform layer of the primate. In work in progress, they find a sequence in which single ganglion cell synapses appear first, followed by single amacrine - amacrine synapses, bipolar ribbon synapses on ganglion cells and finally, bipolar ribbon synapses on amacrine cells. This sequence appears to be genetically determined, because it is not correlated with birth or exposure to light. Ganglion cell dendrites have not been observed to be presynaptic in this study but it will be interesting to use the HRP labelling technique of Sakai et al. to pursue this point.

In Limulus, Fahrenbach speculates that the relatively uniform distribution of output synapses on proximal and distal branches would be determined by the genome during synaptogenesis; by contrast, the distribution of input synapses, heavily skewed toward the proximal branches, might be determined by a gradient of impulse activity spreading decrementally from the axon into the arborization. In this scheme of activity-generated synaptogenesis, "any branch would in effect provide the signal to generate its own inhibitory input, graded with distance from the parent axon" 4 . This would account for the gradation of inhibitory interactions.

How gene expression is synchronized in two or more sets of neurones to bring about the coordinated assembly of synaptic circuits, shaped by concurrent or subsequent functonal activity, is one of the most challenging problems in neurobiology. Microcircuits make excellent test systems for pursuing this problem.

Gordon M. Shepherd is Professor in the Section of Neuroanatomy, Yale University School of Medicine, New Haven, Connecticut 06510, USA.

\title{
Rings and a moon of the planet Uranus
}

On the right are the ten rings of Uranus, photographed from a distance of $1,120,000 \mathrm{~km}$ on 23 January 1986 . This is among the first of the pictures produced by Voyager 2. The newly discovered tenth ring is barely visible about midway between the bright, outermost epsilon ring and the next ring down, called delta. The tenth ring orbits Uranus at a radius of about $50,000 \mathrm{~km}$. On the left is a full-disk view of the moon Titania, taken early in the morning of 24 January 1986 from a distance of $500,000 \mathrm{~km}$. The many cicular depressions are probably impact craters and the bright linear troughs right of the picture are probably canyons. The troughs break the crust in two directions, indicating that the icy satellite has a dynamic, active interior. Photographs courtesy of NASA/Jet Propulsion Laboratory.
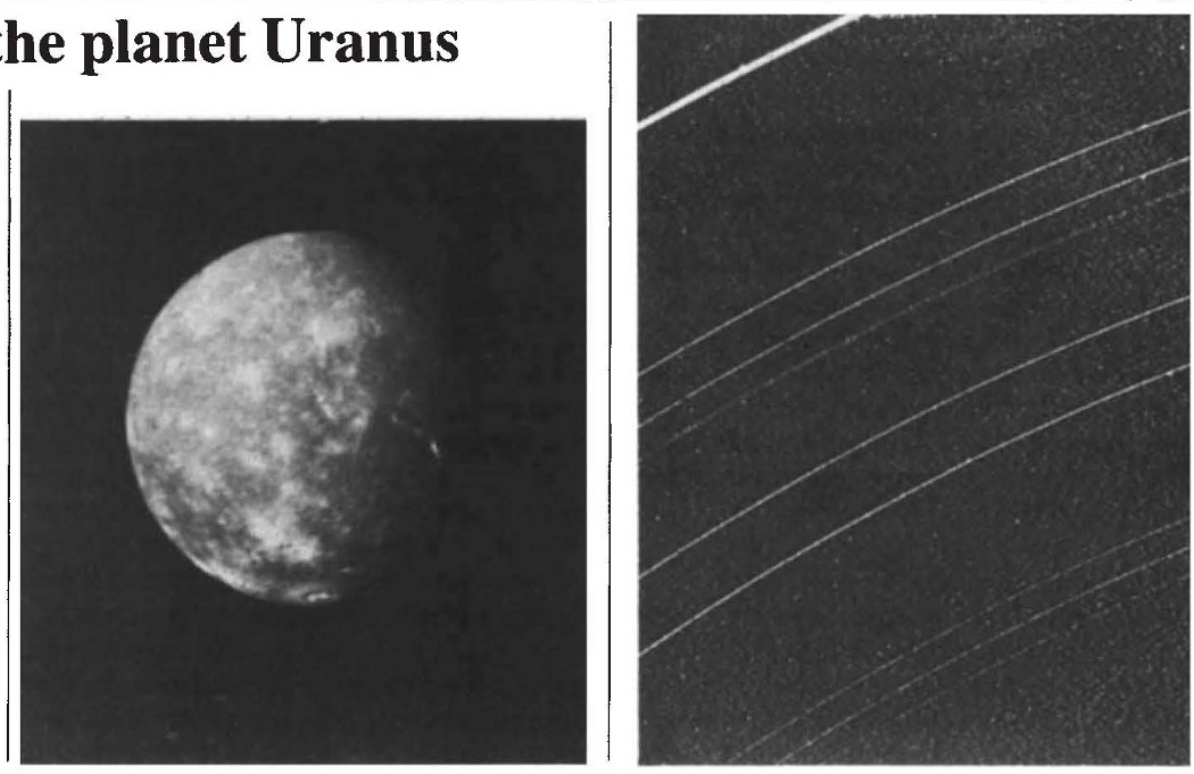